damage are more common and may adversely affect the result is indisputable, but without surgery the outlook is indeed grave. After mitral valvotomy most patients can look forward to many years of improved well-being and increased exercise tolerance.

\section{Summary and Conclusions}

The results are reported of 99 patients aged 50 and over who underwent mitral valvotomy. The immediate mortality was $14(14 \%)$, and a further 11 have died during the period of follow-up, which has varied from six months to nine years. Most of the survivors have benefited from operation and have maintained their improvement even when slight mitral incompetence has been produced. Chronic bronchitis with emphysema, severe pulmonary arterial hypertension, and valve calcification are more common in this age group, and increase both the mortality and the morbidity. Careful supervision is important in the immediate post-operative period.
We wish to thank the many physicians in the region who referred patients for operation.

REFERENCES

Bailey, C. P. (1955). Surgery of the Heart, pp. 544, 639. Kimpton, Belcher, J. R., and Gupta, R. L. (1964). Brit. med. 7., 1, 871.

Dubost, C., Blondeau, Ph., and Piwnica, A. (1962). F. thorac. cardiovasc. Surg., 44, 392.

Fraser, K., and Kerr, I. F. (1962). Brit. med. 7., 2, 443.

Goodwin, J. F., Hunter, J. D., Cleland, W. P., Davies, L. G., and Steiner, R.' E. (1955), ibid., 2, 573.

Logan, A., Lowther, C. P., and Turner, R. W. D. (1962). Lancet, 1, 443.

Lowther, C. P., and Turncr, R. W. D. (1962). Brit. med. F., 1, 1027. Michell, G. (1960). Ibid, 1, 687 .

Mounsey, P. (1957). Ibid., 2, 311.

Obeyesekere, H. I., Dulake, M., Demerdash, H., and Hollister, R. (1965). Ibid., 2, 441 .

Olesen, K. H. (1962). Brit. Heart 7., 24, 349.

Otto, T. J. (1964). Thorax, 19, 541.

Sellors, T. H., Bedford, D. E., and Somerville, W. (1953). Brit. med. Sellors, T. H., Bedford, D. E., and Somerville, W. (1953). Brit. med. Wood, P. 1059. and Spottiswoode, London.

\title{
Viability of Common Pathogens in Cold-curing Acrylic Resin used in Orthopaedic Surgery
}

\author{
A. G. TOWERS,** M.B., B.S., B.SC., M.C.PATH.
}

Brit. med. 7., 1966, 2, 1046-1047

Sokolowski et al. (1955) demonstrated that the acrylic resin monomethyl methacrylate exhibited bacteriostatic activity towards oral micro-organisms. Dutton (1959) drew attention to the ease with which the polymer powder and the powder and liquid dough mix could be contaminated.

Cold-curing acrylic resins with benzoyl peroxide as the catalyst are being used increasingly in orthopaedic surgery, where a tight fit is required between an implant and the bone (e.g., to hold the intramedullary stem of a femoral prosthesis or acetabular replacement). Should infection occur at such a site the result can be most unfortunate for the patient. It is often difficult to remove the resin because of the way it keys into the bone.

Two commonly used products-Surgical Simplex $P$ and Nu-life'-have been examined recently. Surgical Simplex P was available in sterile packs. The powder or polymer is in a double polyethylene pack sterilized by high-energy radiation; the monomer is in ampoules, sterilized by Seitz filtration under positive pressure.

Both components of Nu-life are supplied non-sterile; the powder in 6-oz. (170-g.) plastic bottles and the fluid monomer in 4-oz. (114-ml.) glass bottles. Both kinds of pack favour repeated use and are thus apt to become contaminated.

Oliver and Blaine (1948) stated that acrylic monomer was sterile, but they included a bacterial check on the fluid before use. Charnley (1960) stated that the fluid monomer is self-sterilizing, and that he sterilizes the powder by prolonged exposure to formalin. It is not easy to sterilize the monomer by Seitz filtration under vacuum owing to the ease with which the filter becomes blocked. Judet et al. (1954) stated that some of their patients had a slight serous discharge after the use of acrylic

\footnotetext{
- Department of Biomechanics and Surgical Materials, Institute of Orthopaedics (University of London), Royal National Orthopaedic Hospital, Stanmore, Middlesex

2 Surgical Simplex $P$-Northill Plastics Limited, London. Nu-lifeCottrell and Company Limited, London.
}

prostheses which had been sterilized by formalin. Such discharge had not been seen since the method of sterilization by formalization had been abandoned. This would suggest that there is a possible uptake of formalin by the resin, and that some other method of sterilization of the powder would be preferable.

Curing or hardening of the resin $\mathrm{mix}$ is an exothermic reaction. Peak temperatures in the bone of about $95^{\circ} \mathrm{C}$. may be reached, and it might be thought the temperature and pressure changes which occur with the shrinkage of the resin, coupled with the presence of free monomer, would cause selfsterilization of the preparation.

\section{Results}

The viability of some common pathogens after they had been in contact with these materials was investigated. Tests were made on the powder, monomer, and cured dough with coagulase-positive and coagulase-negative staphylococci, betahaemolytic streptococcus, Bacillus cereus (for spores), Escherichia coli, Proteus vulgaris, and Pseudomonas pyocyanea in 18-hour cultures as the test organisms. Subculture was made at regular intervals to determine what degree of bacterial inhibition could be shown.

The malleable dough was worked well with the fingers, which when "printed" on to blood agar plates gave growth of skin commensals both before and after kneading. Discs of the set resin were also incubated in broth, and $50 \%$ were shown to be contaminated by skin commensals.

Subcultures made from 18-hour broth cultures after intervals of shaking with monomer showed there was a marked variation in survival time for the different test organisms. The haemolytic streptococcus could not be recovered after one minute, the staphylococci varied between 12 and 20 minutes, while the spore-forming $B$. cereus proved the most resistant. Recovery 
of this organism was possible up to 14 days with a two-minute daily shake of the monomer/test-culture mixture. These results confirmed those made by one of the makers (private communication). It is probable that the watery film acts as a protective layer round the micro-organisms, as the broth culture and monomer are immiscible liquids.

Neither monomer dried on filter paper or applied directly in a ditch nor resin discs had any direct inhibitory action towards any of the test organisms when tested routinely. This is at variance with the findings of Sokolowski et al. (1955), who reported "remarkable bacteriostatic activity" with the Czech product Spofacryl.

An 18-hour culture of coagulase-positive staphylococcus scraped from an agar plate direct into monomer resulted in the formation of a brittle residue which was incubated in the monomer for 24 hours at $37^{\circ} \mathrm{C}$. This residue, plated out, gave no growth after 18 hours' incubation, but the residue appeared to be more friable and then spread easily. The test organism was recovered after reincubation for a further period of 18 hours. None of the other test organisms survived in this test. $B$. cereus was not used, as it had shown prolonged survival in broth culture and monomer.

\section{Comment}

These results suggest that some pathogens can remain viable in the cured resin for varying times and to a lesser degree in the monomer, and that for all surgical procedures in the human body only the presterilized material should be used. The monomer should not be put up in rubber-capped bottles, because extraction of the rubber may occur and contamination of the monomer can be introduced, particularly if all the liquid is not used at once. The ampoules should be kept in the dark until required, as sunlight changes the monomer. Ideally the ampoules should themselves be of dark glass. The strictest asepsis should be observed in all the manipulative procedures involved in its use. Two pairs of gloves should be worn by those who knead the resin because of the tensile strains induced in the gloves by the manipulation and the ease with which punctures can be enlarged.

\section{Summary}

The viability of some common pathogens in cold-curing acrylic resin (monomethyl methacrylate) is shown.

Beta-haemolytic streptococci are quickly killed in the fluid monomer; Staphylococcus pyogenes survived 10 to 20 minutes' exposure, while the spore-forming Bacillus cereus remained viable after up to 14 days' exposure.

These resin materials for surgical procedures should be presterilized in suitable quantities for immediate use, and not taken from packs which favour multiple use.

This work has been carried out under the auspices of the Leverhulme Trust Fund.

\section{REFERENCES}

Charnley, J. (1960). F. Bone 7t Surg., 42B, 28.

Dutton, J. (1959). Brit. med. F., 2, 597.

Judet, J., Judet, R., Lagrange, J., and Dunoyer, J. (1954). ResectionReconstruction of the Hip: Arthroplasty with an Acrylic Prosthesis, edited by K. I. Nissen. London.

Oliver, L

Sokolowski, S., Freytag, T., and Kmita, S. (1955). Neurol. Neurochir. Psychiat. pol., 5, p. 253.

\title{
Clinical Trial with Ambilhar in Schistosoma mansoni Infections in Tanzania
}

\author{
J. E. MCMAHON,* M.R.C.S., L.R.C.P. ; C. P. KILALA,* M.B., B.S.
}

Brit. med. F., 1966, 2, 1047-1049

Ambilhar (Ciba 32,644-Ba) is a nitrothiazole derivative, 1-(5nitro-2-thiazolyl)-2-imidazolidinone. Studies have not yet clarified its exact mode of antiparasitic action. It is active only if present in the non-metabolized form, and it is metabolized rapidly by a normal liver.

Promising early reports were published regarding this drug's effectiveness in the treatment of schistosomiasis and amoebiasis. Woodruff (1966) pointed out that the popular press have inferred that the drug is safe for general use, and further " that no paper permitting critical appraisal of the toxicity of the drug in man has yet appeared in a major medical journal."

In this paper we report our findings on its effectiveness as evidenced by follow-up examinations two and four months after completion of treatment, and on observed side-effects during therapy.

\section{Materials and Methods}

Ambilhar was given to 92 African in-patients $(25 \mathrm{mg} . / \mathrm{kg}$. daily in two divided doses for seven days). All the patients were Africans who originated from an area around Mwanza,

* Bast African Institute for Medical Research, Mwanza, Tanzania. on the southern shores of Lake Victoria. Seventy-one returned for the two-month and 63 for the four-month follow-up examinations.

Two different methods were used for detecting eggs in stools: the A.M.S. III technique (Hunter et al., 1948), in which three 1-g. aliquots were examined for Schistosoma mansoni eggs; and the quantitative filtration staining technique (Bell, 1963), which enabled calculation of egg excretion per 24 hours, the sensitivity of the method being sufficient to detect an output of 1,000 eggs per day. Viability of eggs was demonstrated by the hatching test. "Cures" were considered to have occurred only when all three tests were negative.

All patients were clinically examined before, during, and after treatment, and a careful note was made of all side-effects. The majority had pre- and post-treatment electrocardiograms taken.

\section{Results}

The patients were divided into three groups according to pretreatment egg load per 24 hours : group 1, less than 10,000; group 2, 10,000-100,000; and group 3, 100,000-500,000. 